\title{
Modeling proper motions beyond the Galactic bulge
}

\author{
M. Brunetti and D. Pfenniger
}

\author{
Geneva Observatory, University of Geneva, 1290 Sauverny, Switzerland \\ e-mail: maura.brunetti@unige.ch
}

Received 12 November 2009 / Accepted 15 December 2009

\begin{abstract}
We analyse the radial and tangential velocity fields in the Galaxy as seen from the Sun by using as a first approximation a simple axisymmetric model, which we then compare with the corresponding fields in a barred $N$-body model of the Milky Way. This provides a global description of these quantities missing in the literature, showing where they take large values susceptible to be used in future observations even for sources well beyond the Galactic center. Absolute largest proper motions occur at a distance slightly behind the Galactic center, which are there 1.5 times larger than the highest local proper motions due to the Galactic differential rotation. Large proper motions well beyond the Galactic center are well within the current astrometric accuracy.
\end{abstract}

Key words. Galaxy: kinematics and dynamics - Galaxy: general

\section{Introduction}

In view of the ongoing and forthcoming advances of astrometry applied to the Milky Way well beyond the Solar neighborhood, such as the measurements of the trigonometric parallaxes and proper motions of distant stellar masers (e.g., Reid et al. 2009), or the upcoming GAIA survey, we develop here a simple first-order model of a galactic disk making explicit where large proper motions and radial velocities due to differential Galactic rotation can be expected in the Galaxy. It is indeed important to develop quantitative insight on these observables and separate the effects due to differential rotation from those due to, for example, a bar.

The level of analysis is elementary and could figure in astronomy textbooks. Despite literature search and discussions with astrometrists the points raised here do not seem to be commonly known. The probable reason for the lack of a previous similar discussion is that observing proper motions has been considered for a long time as difficult and limited to a few kpc. Further, dust extinction has also limited observations in the Galaxy plane in the optical. Therefore, a local analysis has been considered as sufficient (e.g. Mihalas 1968; Mihalas \& Binney 1981; Binney \& Merrifield 1998).

In radio astronomy, however, the need to develop a global vision of the Galaxy has been reached much sooner. An analysis close to the present one concerning the Galaxy radial velocity field has been already done (see e.g. Burton 1974), since extinction in neutral hydrogen is weak and integrated radial velocity field can be directly measured over most of the Galaxy disk.

The most interesting aspect of the present analysis concerns the proper motion field at distances well beyond the Solar neighborhood, which shows a discontinuous behavior just beyond the Galactic center allowing to measure easily stellar proper motions there and well beyond. The reason is easy to understand if we consider that the disk beyond the Galactic center is moving in first approximation with a velocity opposite to the Sun velocity. The linear velocity difference of about $2 \times 220 \mathrm{~km} \mathrm{~s}^{-1}$ largely exceeds the inversely to distance decreasing proper motions, leading to proper motions up to about $440 \mathrm{~km} \mathrm{~s}^{-1} / 9 \mathrm{kpc} \approx$

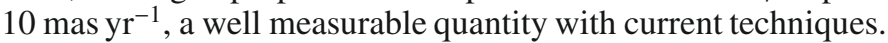

Of course the circular velocity is not equal to the average azimuthal velocity, the velocity that can be observed by averaging proper motions, due to the non-negligible contribution of the velocity dispersion to radial support. Especially close to the Galactic center the velocity dispersion increases to values comparable to $220 / \sqrt{3} \mathrm{~km} \mathrm{~s}^{-1} \approx 130 \mathrm{~km} \mathrm{~s}^{-1}$, therefore one should see, as regions beyond the Galactic bulge are measured at increasing distances, a progressive change from individual random motions at about $130 \mathrm{~km} \mathrm{~s}^{-1} / 8 \mathrm{kpc} \approx 3$ mas $^{-1}$ superposed to a systematic proper motion with respect to an inertial frame due to the Sun motion of about $220 \mathrm{~km} \mathrm{~s}^{-1} / 8 \mathrm{kpc} \approx 5 \mathrm{mas} \mathrm{yr}^{-1}$, progressively increasing to about $400 \mathrm{~km} \mathrm{~s}^{-1} / 10 \mathrm{kpc} \approx 8 \mathrm{mas} \mathrm{yr}^{-1}$ as we explore regions $2 \mathrm{kpc}$ beyond the Galactic center. This insight was already exposed by Binney (1995) in a little read paper concerned primarily with the non-axisymmetric motion effects induced by a bar. He already showed that large proper motions can be expected near the Galactic center even in the absence of a bar. In any case, the systematics introduced by differential motion in the bar/bulge region must not be omitted, as pointed out by Zhao et al. (1996).

We also check that a fully consistent model of the Milky Way including the effects of a bar and velocity dispersions does not change the main conclusions reached with the simple model. For this, we use one of the Milky Way $N$-body models of Fux (1997) including a bar and spiral arms to quantify the differences existing between a fully consistent, self-gravitating, stable and barred model of the Milky Way, and the simple axisymmetric model.

The plan of the paper is as follows. In Sect. 2 we give some definitions. In Sect. 3 we develop the first order axisymmetric model with analytic tools and a more elaborated rotation curve model. In Sect. 4 we analyze Fux's $N$-body model and compare it with the axisymmetric ones and with some observational results. In Sect. 5 we present our conclusions.

\section{Decomposition of a differential velocity field}

Contrary to the tradition, in the following we express formulas as much as possible in Cartesian coordinates in order to avoid 
trigonometric expressions masking simple geometrical interpretation. Bold symbols denote vectors.

For any velocity field $\boldsymbol{V}(\boldsymbol{x}) \in \mathbb{R}^{3}, \boldsymbol{x} \in \mathbb{R}^{3}$ one can define the differential velocity field relative to the velocity of a particular observer located at $\boldsymbol{x}_{0}$, moving at the velocity of the field $\boldsymbol{V}\left(\boldsymbol{x}_{0}\right)$,

$\boldsymbol{v}(\boldsymbol{x}) \equiv \boldsymbol{V}(\boldsymbol{x})-\boldsymbol{V}\left(\boldsymbol{x}_{0}\right)$.

The scalar radial velocity field $v_{\mathrm{r}}(\boldsymbol{x})$ with respect to the observer, the usually observable quantity derived from the Doppler shift, is the projection of $\boldsymbol{v}(\boldsymbol{x})$ along the line of sight unit vector

$\boldsymbol{n}_{\mathrm{r}}(\boldsymbol{x}) \equiv\left(\boldsymbol{x}-\boldsymbol{x}_{0}\right) / d$,

where $d \equiv\left|\boldsymbol{x}-\boldsymbol{x}_{0}\right|$ is the distance to the observer's location. The vector velocity field $\boldsymbol{v}_{\mathrm{r}}(\boldsymbol{x})$ is proportional to $v_{\mathrm{r}}$ and directed in the radial direction $\boldsymbol{n}_{\mathrm{r}}$.

$v_{\mathrm{r}}(\boldsymbol{x}) \equiv \boldsymbol{v}(\boldsymbol{x}) \cdot \boldsymbol{n}_{\mathrm{r}}, \quad \boldsymbol{v}_{\mathrm{r}}(\boldsymbol{x}) \equiv v_{\mathrm{r}}(\boldsymbol{x}) \boldsymbol{n}_{\mathrm{r}}$.

The tangential velocity field is the difference between the velocity field and the radial velocity field,

$v_{\mathrm{t}}(x)=v(x)-v_{\mathrm{r}}(x)$

The observable proper motion field $\mu$ is the tangential velocity field $\boldsymbol{v}_{\mathrm{t}}(\boldsymbol{x})$ divided by the distance $d$ to the observer

$\mu=\frac{\boldsymbol{v}_{\mathrm{t}}(\boldsymbol{x})}{d}$.

This is a 3-dimensional vector field orthogonal to the line-ofsight, tangential to the celestial sphere. It can be converted to two angular velocity components for the desired spherical coordinate system using standard spherical trigonometry formulas. Here we will only use the magnitude of $\mu$.

\section{Axisymmetric models}

The purpose of an axisymmetric model is to provide some insight into rotation curves resembling the Galaxy one. Therefore we choose first a particular representative but simple model of the Milky Way rotation curve that can be handled analytically. In a second step we use a more realistic but still axisymmetric model of the Milky Way (Kalberla 2003).

\subsection{Simple model}

We consider a simple extension of the popular logarithmic axisymmetric galactic potential. We use cylindrical coordinates $(R, z)\left(R^{2}=x^{2}+y^{2}\right.$, with the origin at the Galactic center $x=y=z=0$ ). The potential has the form:

$\Phi(R, z) \equiv \begin{cases}\frac{v_{0}^{2}}{p}\left(1+\frac{R^{2}}{h^{2}}+\frac{z^{2}}{z_{0}^{2}}\right)^{p / 2}, & \text { for } p \neq 0, \\ \frac{v_{0}^{2}}{2} \ln \left(1+\frac{R^{2}}{h^{2}}+\frac{z^{2}}{z_{0}^{2}}\right), & \text { for } p=0,\end{cases}$

where $p$ is a parameter, $p \in[-1,2]$, allowing to study different rotation curves going from an asymptotic Keplerian model $(p=-1)$, to a constant rotation curve $(p \rightarrow 0)$ (the usual logarithmic potential), to an harmonic potential $(p=+2)$. The constants $h$ and $z_{0}$ denote the respective horizontal and vertical scale lengths.

The circular velocity reads

$v_{\mathrm{c}}=\left(\left.R \partial_{R} \Phi\right|_{z=0}\right)^{1 / 2}=v_{0} \frac{R}{h}\left[1+\frac{R^{2}}{h^{2}}\right]^{\frac{p-2}{4}}$.
When $p=0$ the rotation curve $v_{\mathrm{c}}(R)$ is asymptotically flat (for $R \gg h$ ), while for negative/positive values of $p$ it decreases/increases. For $p=2$, it reduces to a straight line, a solid rotation corresponding to a constant density mass model. For $p=-1$ it decreases asymptotically like a Keplerian model where the mass is concentrated at the center. When, additionally to $p=0$, we send $h \rightarrow 0$, the rotation curve becomes a constant $v_{\mathrm{c}}=v_{0}$ for all $R>0$, which corresponds to Mestel's disk rotation curve.

We could have chosen to start directly with a rotation curve expression which represents the effective average azimuthal rotation field. However not any rotation field is consistent with a solution of Jeans' equations and a positive mass distribution. The advantage of the circular rotation curve derived from a potential is that it gives a rotation field consistent with a specific potentialdensity pair, while its drawback is that the circular rotation speed overestimates the effective mean rotation speed.

The circular velocity vector field for a clockwise circular rotation like in the Milky Way reads,

$\boldsymbol{V}(\boldsymbol{x})=-v_{\mathrm{c}} \boldsymbol{n}_{z} \wedge \boldsymbol{n}_{R}=\frac{v_{\mathrm{c}}}{R}\{y,-x, 0\}$,

where $\boldsymbol{n}_{R} \equiv\{x, y, 0\} / R$, and $\boldsymbol{n}_{z} \equiv\{0,0,1\}$ are unit vectors in the galactic plane and perpendicular to it, respectively. The differential vector fields $\boldsymbol{v}(\boldsymbol{x}), \boldsymbol{v}_{\mathrm{r}}(\boldsymbol{x})$, and $\boldsymbol{v}_{\mathrm{t}}(\boldsymbol{x})$, as well as $\boldsymbol{\mu}(\boldsymbol{x})$, can be derived in analytic form using the definitions given above.

The radial vector field reads

$\frac{v_{\mathrm{r}}(\boldsymbol{x})}{v_{0}}=\frac{x y_{0}-y x_{0}}{h d}\left[\left(1+\frac{R^{2}}{h^{2}}\right)^{\frac{p-2}{4}}-\left(1+\frac{R_{0}^{2}}{h^{2}}\right)^{\frac{p-2}{4}}\right]$,

where $d$ is the distance to the Sun. This expression vanishes along the line $x y_{0}=y x_{0}$, and along the circle $R=R_{0}$.

The tangential vector field reads

$$
\begin{aligned}
\frac{v_{\mathrm{t}}(\boldsymbol{x})}{v_{0}}= & \frac{x x_{0}+y y_{0}-R^{2}}{h d}\left(1+\frac{R^{2}}{h^{2}}\right)^{\frac{p-2}{4}} \\
& +\frac{x x_{0}+y y_{0}-R_{0}^{2}}{h d}\left(1+\frac{R_{0}^{2}}{h^{2}}\right)^{\frac{p-2}{4}} .
\end{aligned}
$$

Here the location of vanishing transverse velocity is not simple. $v_{\mathrm{t}}(\boldsymbol{x})$ is symmetric with respect to the line $x y_{0}=y x_{0}$. At the limit $d \rightarrow 0$ the field is proportional to $d$, that is, continuous but not differentiable. This is a well know result from the classical textbook analysis of the local motions around the Sun, where in term of Oort's constants $A=\left.\frac{1}{2}\left(v_{\mathrm{c}} / R-\mathrm{d} v_{\mathrm{c}} / \mathrm{d} R\right)\right|_{R_{0}}$ and $B=$ $-\left.\frac{1}{2}\left(v_{\mathrm{c}} / R+\mathrm{d} v_{\mathrm{c}} / \mathrm{d} R\right)\right|_{R_{0}}$, we have

$v_{\mathrm{t}}=d(A \cos (2 l)+B)$.

This quantity converges to 0 at $d \rightarrow 0$, but with a slope depending on $l$.

For the sake of illustration we show some figures for relevant cases. We set $h=1$ and $p=-0.05$ to study conditions similar to those occurring in our Galaxy for a population of objects with a positive velocity dispersion, which sees its effective rotation decreased by the asymmetric drift. We put the observer at the position $\boldsymbol{x}_{0}=\{8,0,0\}$, which, in kpc unit, corresponds approximately to the Sun position with respect to the Galactic center.

The differential velocity field is shown in the left panel of Fig. 1 and the radial component is shown in the middle panel of Fig. 1 and in the left panel of 2 . Along the $x$-axis, where the observer is placed, the radial velocity vanishes, as well as along the 
M. Brunetti and D. Pfenniger: Modeling proper motions beyond the Galactic bulge
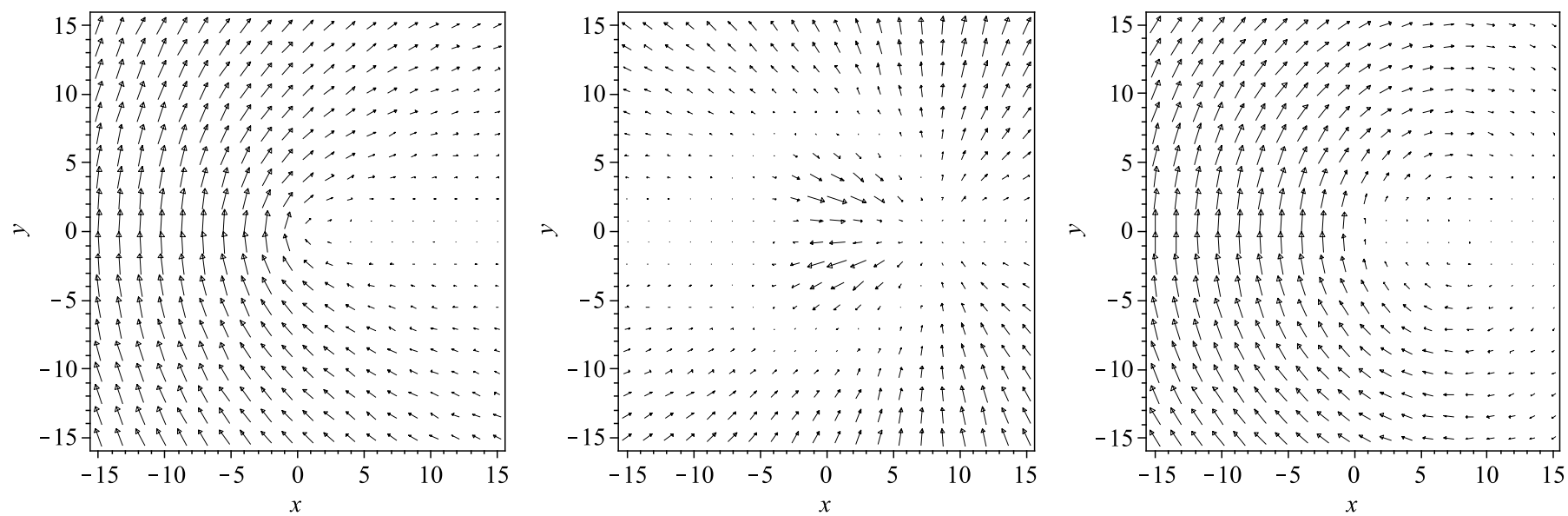

Fig. 1. The differential velocity field for $h=1, p=-0.05, x_{0}=8, y_{0}=0$ (left) and its decomposition in radial (middle) and tangential (right) components.
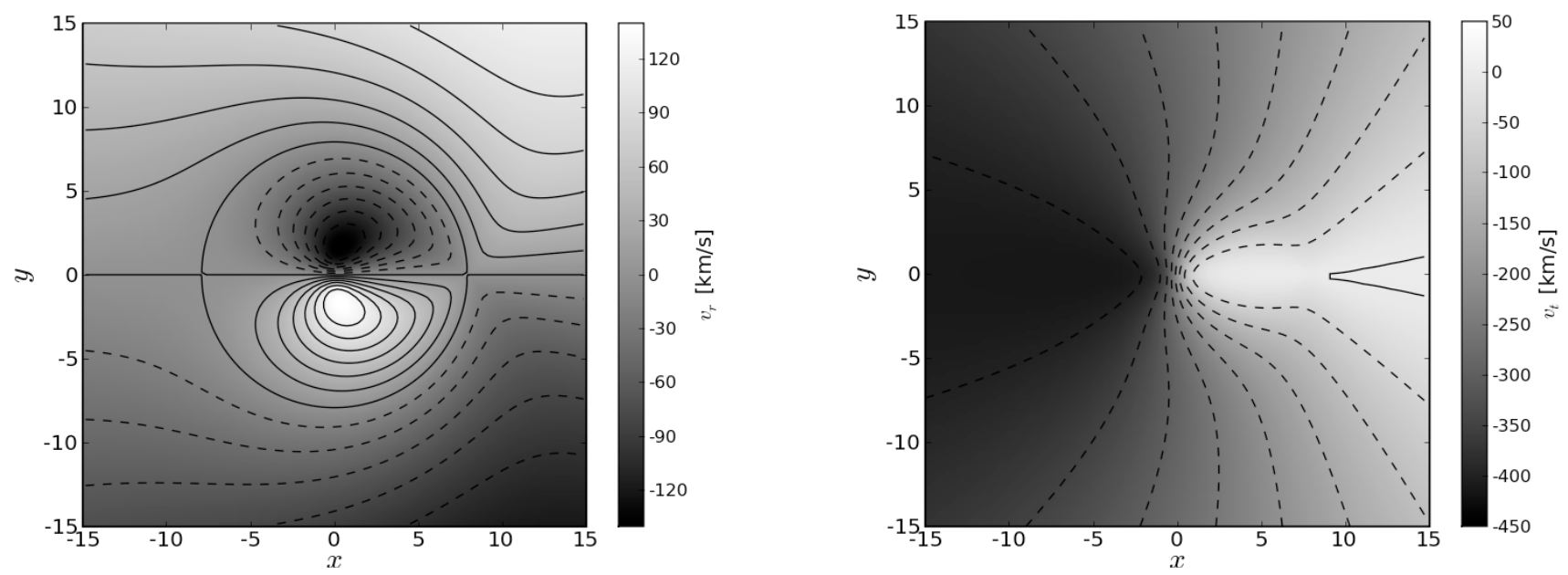

Fig. 2. Contour and gray map of the magnitude of the differential velocity field as in Fig. 1 decomposed in its radial (left) and tangential (right) components. The dark/light grays correspond to low/large values.

circular orbit of the observer at radius $R=8$, as noticed above. The maximum radial velocities within the observer's orbit are located on both sides of the Galactic center direction, and at distances slightly shorter than the Galaxy center. This field has been used extensively in radio astronomy where the neutral hydrogen radial velocity can be measured over most of the Galaxy.

The tangential velocity field is shown in the right panels of Figs. 1 and 2. As can be expected the tangential velocity is small in the direction of the Galaxy center on the same side as the observer, because the matter turns in the same direction at a similar speed there, while the side of the Galaxy beyond the Galactic center sees large tangential velocities because the matter turns in opposite directions. But since only the proper motions are observable, we must consider the proper motion field, shown in Figs. 3.

There is a strongly negative proper motion at the point $(x=-1.395, y=0)$ in a region behind the Galactic center, a region characterized by large amplitude absolute proper mo-

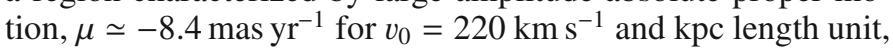
1.5 times larger than the highest local proper motion contribution due to galactic rotation $\left(\mu \simeq-5.6\right.$ mas $\left.r^{-1}\right)$. For positive values of the $x$ coordinate, the proper motion $\mu$ is nearly zero, except at the observer's position where a sharp discontinuity occurs. This follows from the above remark about the local tangential differential velocity close to the Sun (Eq. (11)). Since $\mu=v_{\mathrm{t}} / d$, then $\lim _{d \rightarrow 0} \mu=A \cos (2 l)+B$, i.e., is not single-valued.

The horizontal proper motion as a function of distance along the line Sun-Galactic center takes the following form,

$\mu_{0}(x)=-\frac{v_{0}}{x-x_{0}}\left[\frac{x}{h}\left(1+\frac{x^{2}}{h^{2}}\right)^{\frac{p-2}{4}}-\frac{x_{0}}{h}\left(1+\frac{x_{0}^{2}}{h^{2}}\right)^{\frac{p-2}{4}}\right]$.

The zeros and extrema of $\mu_{0}(x)$ do not have simple analytic expressions. This curve is shown in Fig. 4 (right panel, dashed red line) for a representative choice of the free parameters. Clearly the proper motion maximum amplitude occurs for objects located slightly beyond the Galactic center.

\subsection{Kalberla's model}

Here we check with a more elaborated rotation curve model that our basic result holds, i.e. that the proper motions slightly beyond the Galactic center have largest amplitudes.

We take the model of Kalberla (2003) and extract 40 data points from the rotation curve figure. These data are then represented as a cubic B-spline curve in the symbolic software package Maple 13. This B-spline object can be considered as a usual function on which standard operations, including differentiation, 

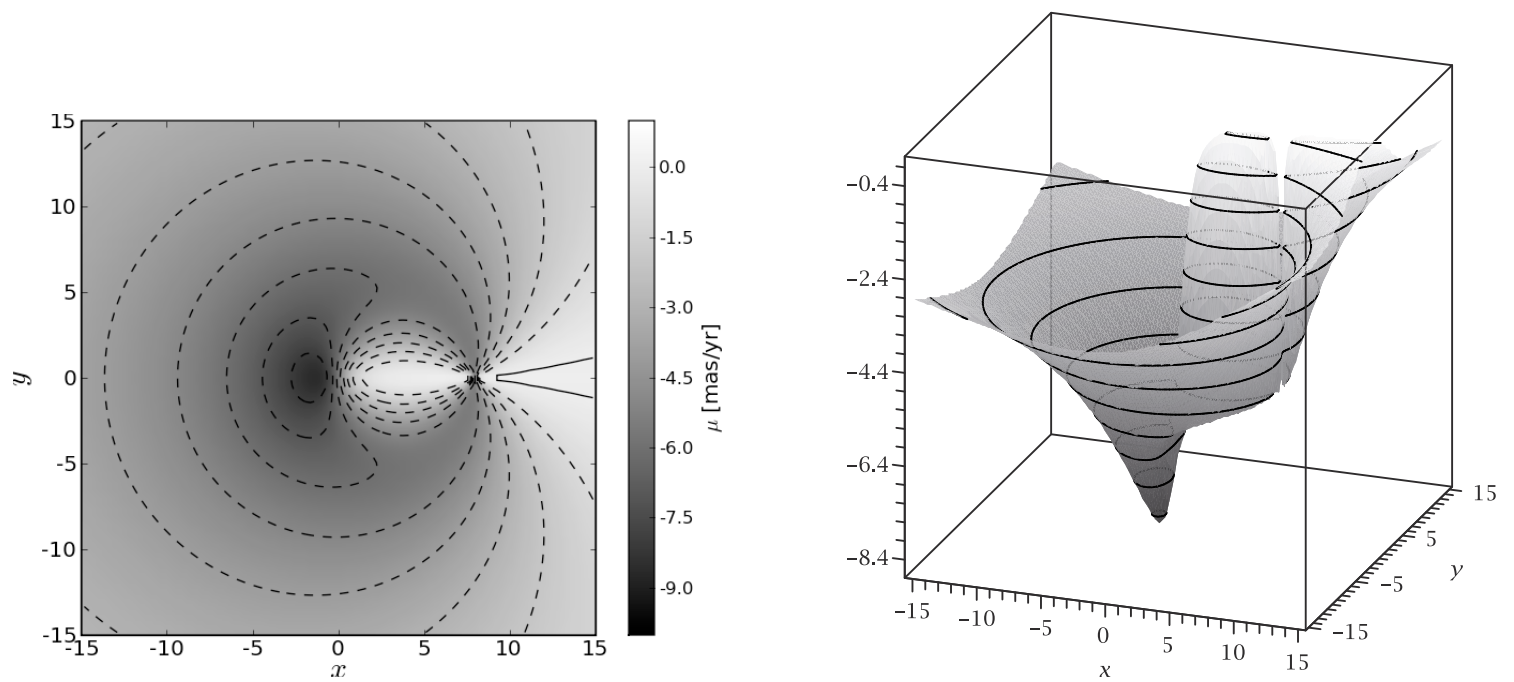

Fig. 3. On the left, contour map of the magnitude of the proper motion $\mu$ as observed from the observer's position at $x_{0}=8, y_{0}=0$ as in Fig. 1 . The dark/light grays correspond to low/large values. On the right, the side view of the same contour map shows the relative importance of proper motion, with the most negative, largest absolute value slightly beyond the Galactic center as seen from the observer's position.
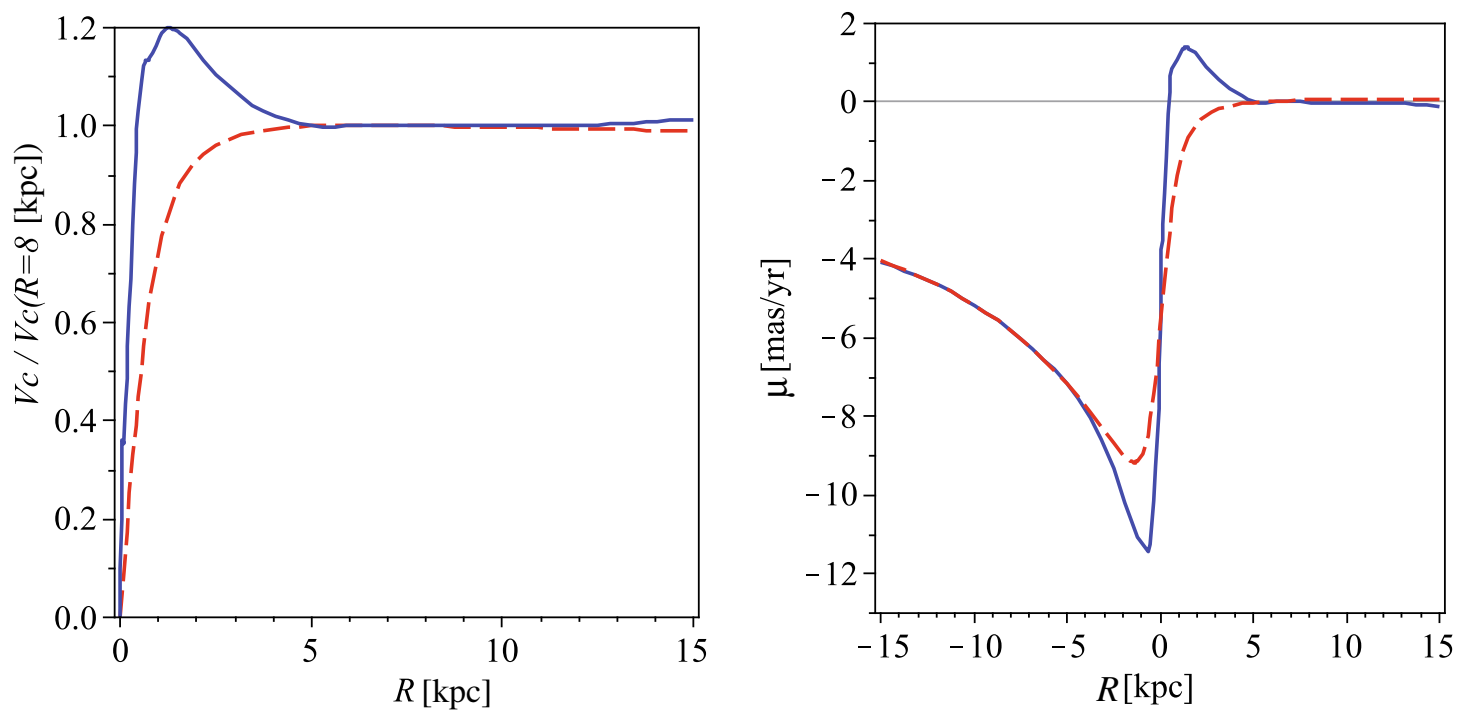

Fig. 4. On the left, rotation curve for the analytic model (dashed red line, same parameters as in Fig. 1) and the Kalberla model (solid blue line). On the right, proper motion $\mu_{0}$ along the line passing through the Galactic center as observed from the observer's position at $R_{0}=8$, in mas yr ${ }^{-1}$ for $v_{0}=220 \mathrm{~km} \mathrm{~s}^{-1}$ and kpc length unit, for the analytic model (dashed red line, same parameters as in Fig. 1) and the Kalberla model (solid blue line).

can be applied. The analysis can be repeated exactly as for the previous model.

Since the results turn out to be qualitatively the same, we only show the initial rotation curve and the final proper motion curve along the direction of the Galactic center in Figs. 4 (solid blue lines). The main difference occurs near the center inside the inner $3 \mathrm{kpc}$ where the rotation curve presents a bump and a steeper raise. This feature may be due to the streaming motions in the bar and, for applying it to observed stars, it should be corrected from the large velocity dispersion there by decreasing the average circular motion. Thus the higher proper motion amplitude (up to 12 mas $\mathrm{yr}^{-1}$ ) obtained here beyond the Galactic center is probably an upper bound of what could be observed.

The differences between the models are largest precisely in the bulge-bar region where the absolute proper motions are largest, i.e., easier to obtain. Proper motions therefore represent a powerful observable for discriminating models of the bulgebar kinematics.

\section{Barred $N$-body model}

The previous models based on rotation curves do not take into account the decrease of average rotation due to asymmetric drift, nor the effects of a bar. Both effects are largest in the bulge-bar regions, therefore it is useful to compare our previous models to a fully self-consistent model of the Milky Way including a bar.

Here we analyse one of the barred models of the Milky Way obtained by Fux (1997) and we compare it with the axisymmetric models discussed in the previous section and with observational results.

Fux's barred models result from the self-consistent $N$-body evolution of a bar-unstable axisymmetric model, with $10^{5}$ particles in the nucleus-spheroid component (which is well suited to represent the nuclear bulge and the stellar halo) and in the disk component, and $10^{5}$ particles in the dark halo component (which has been added to ensure a flat rotation curve at large radii). The spatial location of the observer has been constrained by 


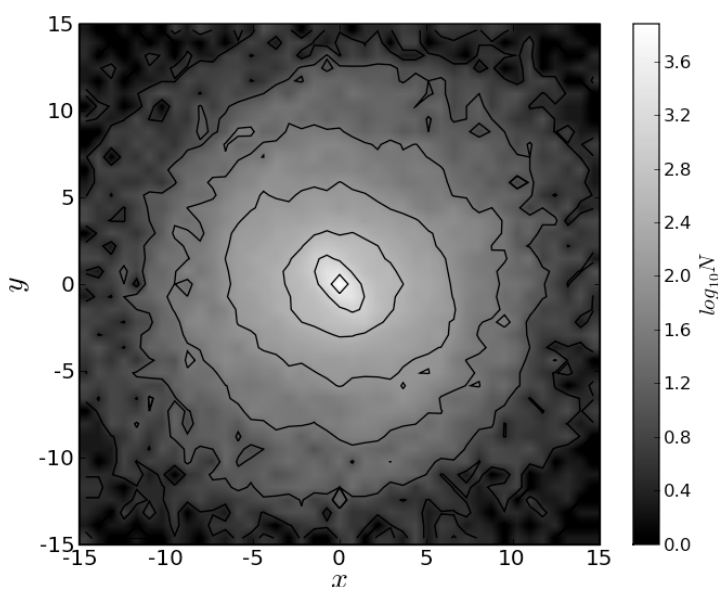

Fig. 5. Density distribution for the barred $N$-body model m $08 \mathrm{t} 3200$.

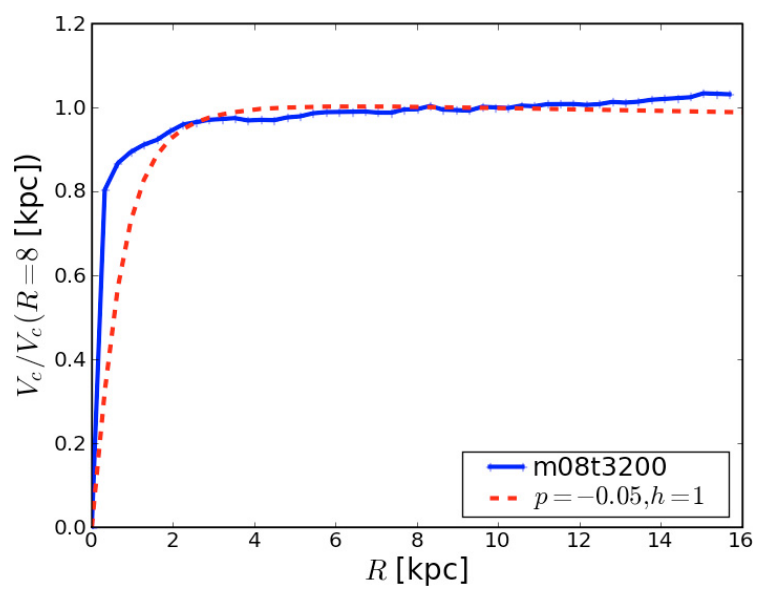

Fig. 6. Rotation curve for the barred $N$-body model m08t3200 (solid blue line) and the analytic model (dashed red line, same parameters as in Fig. 1).

the $K$-band map, obtained by the Diffuse Infrared Background Experiment (DIRBE) on board the COBE satellite and corrected for extinction by dust, while masses have been scaled according to the observed radial velocity dispersion of M giants in Baade's window. Among the models obtained by imposing such constraints, the model named m08t 3200 is recommended in Fux (1997) since it reproduces satisfactorily the kinematics of disk and halo stars in the Solar neighborhood.

The density distribution of m $08 \mathrm{t} 3200$ is shown in Fig. 5. By a rotation of the particles the observer has been moved to the position $\boldsymbol{x}_{0}=\{8,0,0\}$ in kpc unit. The angle $\phi$ between the line joining the observer to the Galactic center and the major axis of the bar equals to $\phi=25^{\circ}$ and the corotation radius is $R_{L}=$ $4.8 \mathrm{kpc}$ (Fux 1997).

The rotation curve is shown in Fig. 6, solid blue line. For comparison, we also show the rotation curve for the analytic model discussed in the previous section, for $p=-0.05, h=1$. As in Kalberla's model, the main difference occurs near the central $3 \mathrm{kpc}$, with the absence of a bump.

The differential velocity field $v$ defined in Eq. (1) is now obtained by averaging the particle velocities $\boldsymbol{V}_{i}$ located at different $z$ positions to smooth out irregularities due to granularity, and by subtracting the averaged velocity vector $\overline{\boldsymbol{V}}\left(\boldsymbol{x}_{0}\right)$ of the particles in a volume of $1 \mathrm{kpc}^{3}$ centered at the position of the observer $\boldsymbol{x}_{0}$. The radial velocity field and the tangential velocity field are shown in Fig. 7 (top and bottom panels, respectively)
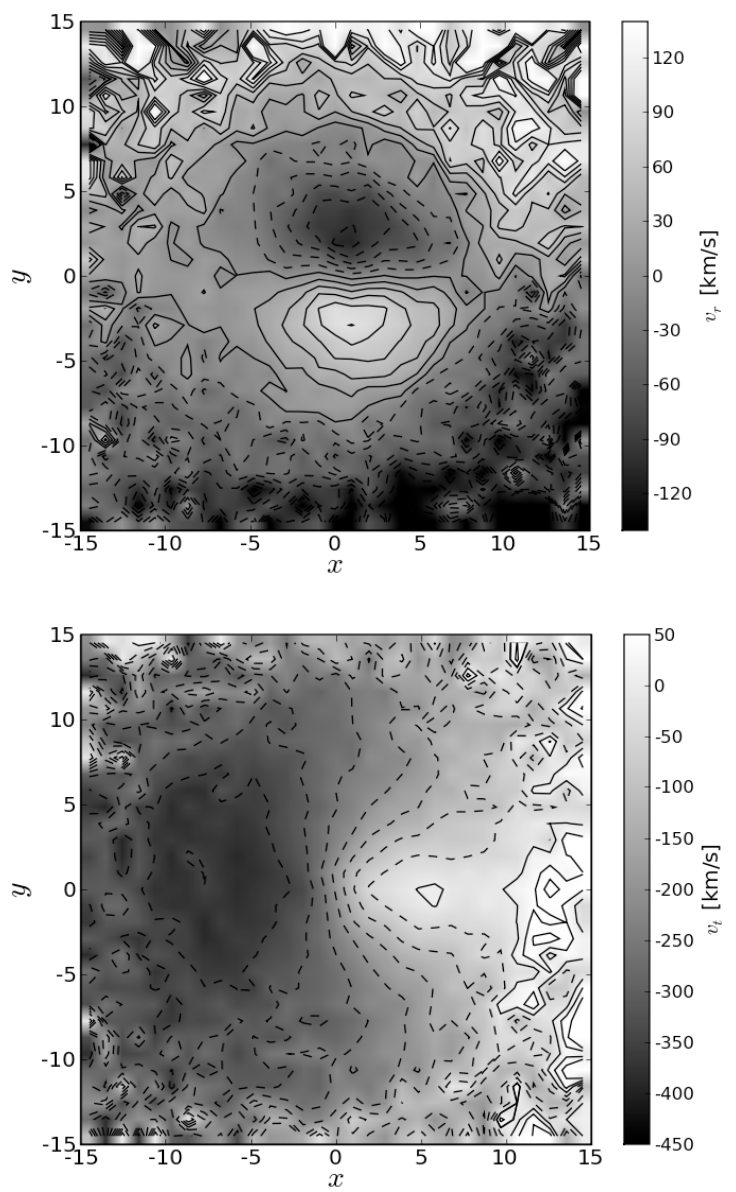

Fig. 7. Contour map of the magnitude of the differential velocity field for the barred $N$-body model m08t3200 decomposed in its radial (top) and tangential (bottom) components.

and should be compared with the fields shown in Fig. 2 for the analytic axisymmetric model. From these panels it can be seen that, apart from noise in the boundary cells due to the finite number of particles, the overall structure of the contour maps is in agreement with those corresponding to the analytic model. The presence of the bar does not introduce asymmetries changing the overall results. In order to quantify the effect of the bar, we have constructed a symmetric system by randomising the particle polar angles. Thus, we could evaluate that the relative error in both radial and tangential velocity fields due to the presence of the bar is less than $15 \%$.

The proper motion field (averaged on $z$ ) and the proper motion $\mu_{0}$ (averaged on $z$ and $|y|<1 \mathrm{kpc}$ ) are shown in Fig. 8 (left and right panels, respectively). Also in this case the overall structure of the contour map agrees with the analytic model map (compare with Figs. 3) and the behavior of $\mu_{0}$ is analog to that shown in Fig. 4 (right panel) apart from numerical oscillations due to the presence of the singular point at the observer's position. The relative error between the barred model and the one obtained by randomising angles is $6 \%$. As in the axisymmetric models, in Fux's barred model a region of particles with large-amplitude proper motion exists, located behind the Galactic center. In this region, the proper motion is of the or-

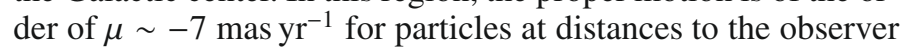
of $10 \mathrm{kpc}$. The same order of magnitude holds in regions slightly above and below the Galactic plane, which can be reached by direct measurements. This order of magnitude for the proper motion is well within the astrometric accuracy of the GAIA survey, 

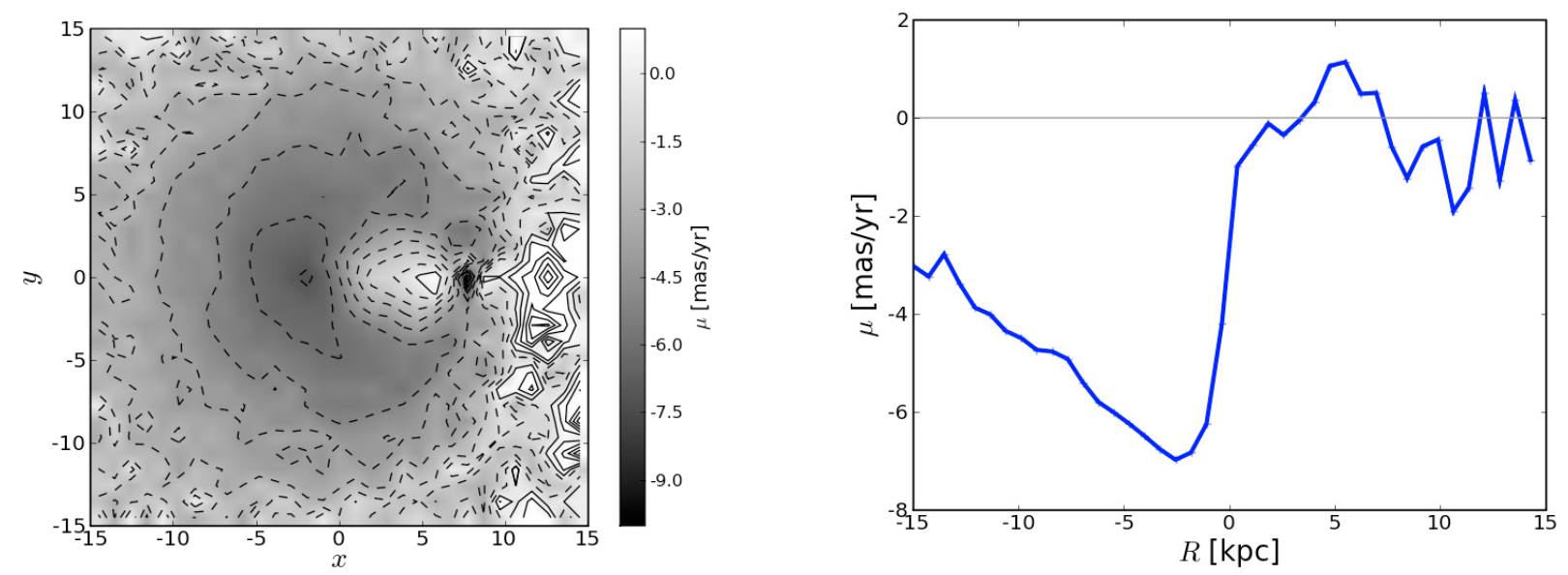

Fig. 8. Contour map of the magnitude of the proper motion $\mu$ as observed from the observer's position at $x_{0}=8, y_{0}=0($ left $)$ and proper motion $\mu_{0}$ along the line passing through the Galactic center (right) for the barred $N$-body model m $08 \mathrm{t} 3200$.
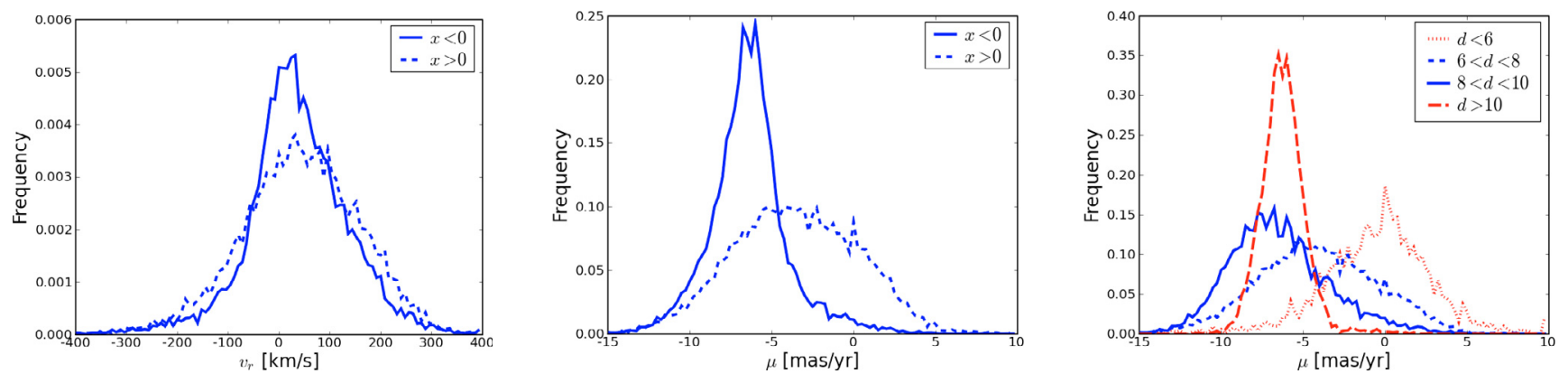

Fig. 9. Distributions of radial velocities (left) and proper motions (middle) obtained by selecting stars with $0<l<20^{\circ}$ and positive/negative $x$ in the barred $N$-body model m08t3200. Right panel: proper-motion distributions obtained by selecting stars with $0<l<20^{\circ}$ and different distance $d$ from the observer.

which will be 12-25 $\mu$ as at the 15 th magnitude and 100-300 $\mu$ as at the 20th magnitude (see e.g. Brown 2007).

We compare now the predictions of this $N$-body model with some observational results in order to understand how the parameters of the Galactic bar can be constrained by measurements of radial velocities and proper motions. Mao \& Paczyński (2002) used two simple analytic models to show that samples of stars in the far and near sides of the bar display differences in both radial and tangential streaming motions. In that paper, it was suggested to use red clump giants to constrain the kinematics of stars in the Galactic bar. Red clump giants are bulge stars which occupy a distinct region in the color-magnitude diagram. They have a well-defined peak in their observed luminosity function, which can be used to select stars at the bright slope of the peak, and thus closer to us, from those at the faint slope which are more distant. Following this suggestion, data of the Optical Gravitational Lensing Experiment II (OGLE-II) in the Baade's window were used to derive the difference in the average proper motion between the bright and faint samples of red clump giants (Sumi

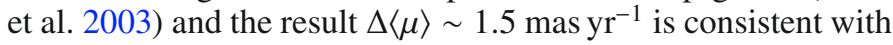
the value estimated in Mao \& Paczyński (2002) who assumed a streaming motion of the bar of $v \sim 100 \mathrm{~km} \mathrm{~s}^{-1}$ in the same direction as the solar rotation.

More recently, different methods were used in order to select bulge samples. Vieira et al. (2007) measured proper motions inside Plaut's window with an accuracy of 1 mas yr $^{-1}$ using ground-based data. They selected a bulge sample at the mean distance of $6.37 \mathrm{kpc}$ in the red giant branch by cross-referencing with the Two Micron All Sky Survey catalog. Clarkson et al. (2008) reported on the use of the Advanced Camera for Surveys
WFC on the Hubble Space Telescope to extract proper motions in the Sagittarius window with an accuracy of 0.3 mas $\mathrm{yr}^{-1}$. They selected kinematically a bulge sample by introducing a cutoff on longitudinal proper motions and on proper-motion measurement errors, as suggested by Kuijken \& Rich (2002). An interesting result of such studies is that longitudinal proper motions can clearly separate near and far samples of stars.

We use Fux's accurate model in order to evaluate the effect of distance on radial-velocity and proper-motion measurements. We consider almost 24000 particles with Galactic longitude $0<l<20^{\circ}$ and we compare their distribution in the near region $x>0$ with that in the far region $x<0$, averaged on $|z|<0.5 \mathrm{kpc}$. The distributions of radial velocities $v_{\mathrm{r}}$ and proper motions $\mu$ are shown in the left and middle panels of Fig. 9, respectively. From the left panel, it can be seen that the far sample in $x<0$ is slightly shifted toward more negative radial velocities, in qualitatively agreement with Mao \& Paczyński (2002). The shift between the two samples is $\Delta\left\langle v_{\mathrm{r}}\right\rangle \sim 5.4 \mathrm{~km} \mathrm{~s}^{-1}$. Longitudinal proper motions show much clearer association with distance than radial velocities. The middle panel in Fig. 9 shows that the distribution of far-sample proper motions is shifted toward more negative values with respect to that of the near sample, with a

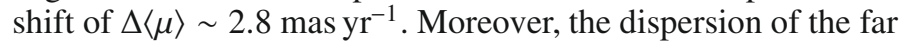
sample is $60 \%$ smaller than that of the near sample.

In order to discuss the effects of contamination of bulge samples by disk stars we select stars in Fux's model with different mean distances from the observer. Since the corotation radius is $R_{L}=4.8 \mathrm{kpc}$ and axis ratio is $b / a=0.5$ in Fux's model, samples in $0<l<20^{\circ}$ and $6<d<10$ (in kpc length unit) consist mainly of bulge stars, while those in $d<6$ or $d>10$ have 
large contamination by disk stars. In the right panel of Fig. 9 we show the proper-motion distributions obtained by selecting stars within these regions. By comparing the right and middle panels of Fig. 9, we see that the effect of the contamination from distant $(d>10)$ and nearby $(d<6)$ disk stars is to move the mean value of the bulge samples toward more positive values. Distant disk stars also have the effect of reducing the dispersion of bulge proper-motion distribution, as discussed in Vieira et al. (2007). Knowledge of the global distribution of proper motions as shown in Figs. 3 and 8 help us to understand and to evaluate these effects of contamination.

\section{Conclusions}

Starting from the analysis of simple axisymmetric models of the Milky Way, we have studied the differential velocity field, and in particular the proper motion field, providing a global description of observables. Interestingly, there is a region behind the Galactic center extending to several kpc further out where the proper motions induced by differential rotation have the largest amplitudes in all the Milky Way, an apparently little known basic feature (with the exception of Binney1995) with direct observational consequences. Here we clarify on a global scale the effects related to first order to the average circular rotation and to second order to a bar.

We have checked that these high proper motions are also found in self-consistent barred configurations, such as the Milky Way $N$-body model of Fux (1997). The proper motions at distances of $10 \mathrm{kpc}$ to the observer are of the order of 7 mas yr$^{-1}$ and thus are well within the accuracy of the forthcoming surveys such as GAIA. It is crucial to be able to separate the sources according to distance, at least to separate the sources closer to or beyond the Galactic center.
Provided that the position of the sources, such as stars or $\mathrm{OH}$ masers, are intense enough to be measured with respect to an absolute frame of reference, as well as their radial velocities, the global velocity field of the Milky Way including non-circular motions should become accessible. This will be a first order information for constraining the strength of the bar and the mass distribution in the bulge, the keystone of the whole Galaxy.

Acknowledgements. We thank Roger Fux for providing us the simulation data and Laurent Eyer for useful discussions. We thank James Binney for constructive comments. This work has been supported by the Swiss National Science Foundation.

\section{References}

Binney, J. 1995, in Astronomical and astrophysical objectives of submilliarcsecond optical astrometry, ed. E. Hog, \& P. K. Seidelmann (Dordrecht: Kluwer), IAU Symp., 166, 239

Binney, J. J., \& Merrifield, M. 1998, Galactic Astronomy (Princeton University Press)

Brown, A. G. A. 2007, Getting ready for the microarcsecond era, in A Giant Step: from Milli- to Micro-Arcsecond Astrometry, ed. W. J. Jin, I. Platais, \& M. A. C. Perryman, Proc. IAU, 3, 567

Burton, W. B. 1974, Galactic and Extra-galactic Radio Astronomy, ed. G. K. Verschur, \& K. I. Kellermann (New York: Springer), 82

Clarkson, W., Sahu, K., Anderson, J., et al. 2008, ApJ, 684, 1110

Fux, R. 1997, A\&A, 327, 983

Kalberla, P. M. W. 2003, ApJ, 588, 805

Kuijken, K., \& Rich, R. M. 2002, ApJ, 124, 2054

Mao, S., \& Paczyński, B. 2002, MNRAS, 337, 895

Mihalas, D. M. 1968, Galactic Astronomy (San Francisco: Freeman)

Mihalas, D. M., \& Binney, J. J. 1981, Galactic Astronomy, 2nd edn. (San Francisco: Freeman)

Reid, M. J., Menten, K. M., Zheng, X. W., et al. 2009, ApJ, 700, 137

Sumi, T., Eyer, L., \& Woźniak, P. R. 2003, MNRAS, 340, 1346

Vieira, K., Casetti-Dinescu, D. I., Méndez, R. A., et al. 2007, ApJ, 134, 1432

Zhao, H., Rich, R. M., \& Biello, J. 1996, ApJ, 470, 506 